\title{
CHANGES IN SOME PERIPHERAL BLOOD IMMUNE PARAMETERS OF PATIENTS WITH CHRONIC PERIODONTITIS AMONG SERVICEMEN OF THE ARMED FORCES OF UKRAINE
}

\author{
Pavlo Schmidt ${ }^{1}$, Anatoliy Gulyuk ${ }^{2}$ \\ 'National Military Medical Clinical Center "Main Military Clinical Hospital", Kyiv, Ukraine, \\ hochadler@ukr.net \\ ORCID: http://orcid.org/0000-0001-7449-3512 \\ ${ }^{2}$ Department of Surgical Dentistry, Odessa National Medical University, Odessa, Ukraine \\ ORCID: http://orcid.org/0000-0003-3953-5339
}

\begin{tabular}{l} 
ARTICLE INFO \\
\hline Article history: \\
Received date 23.09 .2020 \\
Accepted date 26.10 .2020 \\
Published date 30.10 .2020 \\
Section: \\
Practical medicine \\
DO I
\end{tabular}

$10.21303 / 2313-8416.2020 .001461$

KEYWORDS

periodontitis

periostitis

immunogram

immune status

\section{ABSTRACT}

The object of research: the state of chronic periodontitis among servicemen of the Armed Forces of Ukraine remains extremely urgent. Therefore a group of servicemen with chronic periodontitis and its complications was taken. In addition, a control group of servicemen was used to compare the results.

Investigated problem: in this article, the diagnosis was considered due to the use of immunological studies of peripheral blood of sick servicemen of the Armed Forces of Ukraine with chronic periodontitis, as well as its complications. The study of the features of immunological parameters in peripheral blood was performed.

The main scientific results: according to the obtained data, there is a relative suppression of immunity among servicemen with chronic periapical infection compared with servicemen of the control group.

According to our data, the main lymphocyte subpopulation of peripheral blood are CD8 and CD4/CD8; CD19+(B-lymphocytes) and CD3-HLA+; CD4 and CD4/CD8, which reflect inflammatory processes in the body. These indicators are closely interrelated.

The area of practical use of the research results: these results of research can be applied to students' training at stomatology faculties, in stomatology practice, to research into the immunological status of patients with chronic periodontitis and its complications.

Innovative technological product: it is the first time the study of the immunological status of peripheral blood during chronic periapical infections among servicemen has been performed.

Scope of the innovative technological product: this study can be used in the diagnosis and treatment of chronic periodontitis in dental practice, as well as in the research to compare the results of the immunological state of peripheral blood.

(C) The Author(s) 2020. This is an open access article under the CC BY license http://creativecommons.org/licenses/by/4.0).

\section{Introduction}

\section{1. Object of study}

In this work we conduct a study of servicemen of the Armed Forces of Ukraine with chronic periodontitis and their complications. The state of chronic periodontitis among servicemen of the Armed Forces of Ukraine remains extremely urgent [1]. This pathology, according to the literature and our observations, ranks third in visits to the dentist [2].

The topical problem of dentistry is the prevention, diagnosis and treatment of periodontal tissue diseases, which are the cause of premature tooth losses, secondary adentia, leads to impaired speech, masticatory function and, in addition, are the risk of pathological processes not only in tissues and organs of the mouth, but also general somatic disorders due to the presence of odontogenic foci of infection [3].

To improve the diagnosis, the immune status of patients' peripheral blood was studied. It is determined which marker responds better and more clearly indicates inflammatory processes in the body as a marker of inflammation in chronic periodontitis. 


\section{2. Problem description}

Apical periodontitis is an inflammatory process induced by necrosis of the pulp and subsequent entry of infected and toxic detritus from the root canal system of the teeth through the apical hole into the apical anatomical structures, which ranks third in visits to the dentist. The results of research in recent years show the presence of a number of immunohistochemical criteria that can predict the course of the destructive process in periodontal tissues. To assess the level of antimicrobial protection of the body, the determination of non-specific factors and immunological parameters is performed [4].

Thus, reliable and adequate diagnosis, prediction of the disease and effective treatment of chronic apical periodontitis is impossible without conducting and analyzing standardized and improved immunological studies.

\section{3. Suggested solution to the problem}

According to the results of a number of studies among dental diseases, periodontitis ranks third after caries and pulpitis in the age group of 25-47 years. The inflammatory process in the periodontium as a result of pulp necrosis leads to the initiation of cascade reactions at the cellular, immune and microcirculatory levels, resulting in the destruction of periodontal tissues. The results of research in recent years show the presence of a number of immunohistochemical criteria that can predict the course of the destructive process in periodontal tissues. To assess the level of antimicrobial protection of the body, the determination of non-specific factors and immunological parameters was performed [5].

According to research among patients with chronic apical periodontitis, there is suppression of nonspecific resistance in the form of a decrease in the amount and activity of lysozyme, immunoglobulins and impaired cytokine status $[6,7]$. The dental status of patients, as well as the effectiveness of treatment carried out in case of pathological process in periapical tissues, significantly depends on the state of nonspecific resistance of the organism, in particular, in the oral cavity provided by the coordinated work of neuroendocrine, immune, bactericidal, antitoxic, antioxidant, protease-inhibitory and other systems [8].

Thus, reliable and adequate diagnosis, prediction of the disease and effective treatment of chronic apical periodontitis is impossible without the conduct and analysis of standardized and improved immunological studies.

The main aim of the work is to study the features of immunological parameters of peripheral blood among sick servicemen of the Armed Forces of Ukraine with chronic periapical infection.

\section{Materials and methods}

Immunograms have been performed in the research laboratory of experimental oncology of the National Cancer Institute.

All patients underwent hospital treatment at the National Military Medical Clinical Center "NMMCC" in 2018-2019. During the treatment, they had immunograms performed. All studies are regulated by the Commission on Bioethics №104 of 14.05.2020 at the State Institution "Institute of Dentistry and Maxillofacial Surgery of the National Academy of Medical Sciences of Ukraine" and in compliance with the Code of Ethics of the World Medical Association (Declaration of Helsinki).

The study of peripheral blood cellular immunity has been conducted on 36 (100.0\%) patients in the armed forces. Depending on the clinical course of periapical periodontitis, patients were divided into two groups. To detect changes in immunocompetent cells during the complication of chronic periapical infection, the largest number of subjects was group I, which received $31(86.1 \%)$ patients; control group - group II, consisted of 5 (13.9\%) patients without signs of acute or chronic inflammation (we assessed it as asymptomatic).

Student's method has been used for statistical calculations. The data in the tables are presented in absolute numbers (abs.), fractions in \% (P) and fraction error $(\mathrm{P} \pm \mathrm{Sp})$.

All fluorescent label detection studies are performed on a FACS Calibur (Becton Dickinson, USA) equipped with two lasers (488 and $625 \mathrm{~nm}$ wavelengths). CellQuest-PRO for Macintosh computers has been used to obtain and analyze the data. A narrowband 642/75 nm filter has been used to measure fluorescence. 
The stages of preparation of samples for flow cytofluorometry are based on the following:

1. Put $5 \mu$ l. monoclonal antibodies (CD3, HLA-DR, CD20, CD 4, CD 8, CD16, CD95, CD11b. CD38) into dry cytometric tubes, do not put antibodies into the control (add $5 \mu 1$ of isotype control);

2. Put $20 \mu$ l. of heparinized blood in these tubes;

3. Incubate for 30 minutes in the dark;

4. Add $10 \mathrm{ml}$. of hemolytic fluid;

5. Incubate for 10 minutes in the dark;

6. Spin in a centrifuge for 10 minutes at $1500 \mathrm{rpm}$;

7. Drain the supernatant and add $2 \mathrm{ml}$ of PBS

8. Spin in a centrifuge for 10 minutes at $1500 \mathrm{rpm}$;

9. Drain the supernatant and add $400 \mu \mathrm{l}$ of $0.4 \%$ formalin;

10. Further analysis is performed on a FACS Salibur laser flow cytometer (Becton Dickinson, USA) $[9,10]$.

\section{Results}

According to the laboratory study of the main subpopulations of peripheral blood lymphocytes, the average $\mathrm{CD} 3+$ cells in the blood of servicemen with chronic periodontitis were within normal limits (56-82\%), was 59.4 $\pm 6.0 \%$.

Average figures CD19+cells (B lymphocytes) in the main group were within normal limits $(6-12 \%)$, amounting to $10.0 \pm 5.8 \%$. The decrease in this indicator in the absence of congenital pathology and removed spleen can be observed in acute viral and chronic bacterial infections. A significant difference has been found in the CD 19+cell values between the main and control groups; in the main group, the average value of this indicator was less than in the control group $(16.6 \pm 1.9 \%)$ by $6.6 \%$, or in 1.66 times $(\mathrm{p}=0.019)$; this difference can be considered as significant. The relative decrease in B-lymphocytes indicates a lower involvement of humoral immunity in response to chronic periapical infection than in the control. The average value of CD 19+in the control group exceeded the upper limit of normal (12\%) slightly by $27.7 \%$, or 1.3 times.

As a consequence of chronic inflammation, the average values of CD4+in the main group were 1.27 times, and in the control group - 1.1 times (i. e. slightly) below normal (38.0-43.0 \%), amounting to $29.8 \pm 7.3$ and $34.2 \pm 7.0 \%$, respectively $(\mathrm{p}=0.2)$.

The average values of CD $8+$ cells in the blood of patients in both groups were higher than normal $(19.0-24.0 \%)$; this figure in the main group was significantly higher than in the control: $28.8 \pm 7.5 \%$ vs. $25.3 \pm 5.6 \%$, respectively $(\mathrm{p}<0.05$ ) (Table 1 ), which is an indicator of greater severity of suppressive activity of cellular immunity in chronic periapical infection.

Table 1

Average figures CD8+(T-cytotoxic suppressors) of blood

\begin{tabular}{cccc}
\hline \multirow{2}{*}{ Indicators (norm 19-24 \%) } & \multicolumn{2}{c}{ Research groups } & p \\
\cline { 2 - 3 } & Main group $(\mathbf{n}=\mathbf{3 1})$ & Control group (n=5) & \\
\hline $\mathrm{M} \pm \mathrm{m}$ & $28.8 \pm 7.5^{*}$ & $25.4 \pm 5.6^{*}$ & 0.019 \\
$\mathrm{Me}\left[\mathrm{Q}_{1} ; \mathrm{Q}_{2}\right]$ & $30[7 ; 14]$ & $27[25 ; 29]$ & $25.4[18.5 ; 32.3]$
\end{tabular}

Note: the variety of values in the main and control groups are statistically significant when $p<0.05$

Both in the main group and in the control, the average values of CD3-CD16+CD56+(NK-killers) were within normal limits $(10.0$ - 16.0 \%), amounting to $15.9 \pm 7.3$ and $13.0 \pm 4.0 \%$, respectively $(\mathrm{p}=0.39)$, decreased (below $10.0 \%$ ) values of natural killer cells (CD3-CD16+CD56+) were determined in $16.1 \pm 6.6 \%(n=5)$ of patients in the main group and in $20.0 \pm 17.9 \%(n=1)$ control group ( $\mathrm{p}>0.05$ ). The average value of decreased low CD3-CD16+CD56+in the main group was $6.8 \pm 1.2 \%$, which was 1.47 times less than normal. In the control group there was 1 case of decrease CD3-CD16+CD56+to $9 \%(\mathrm{p}>0.05)$. 
The average values of CD3+CD16+CD56+were higher than normal (2.0-5.0 \%) both in the main group (significantly - 2.1 times) and in the control one (1.4 times): $10.8 \pm 7.6 \%$ vs. $7.0 \pm 2.7 \%$, respectively, $\mathrm{p}=0.27$.

A slight decrease in the average value of $\mathrm{CD} 4 / \mathrm{CD} 8$ in the main group to 1.09 , which is below the lower limit of normal (1.3) by 1.2 times, cannot be taken as a state of immunosuppression, however, the relative suppression of immunity among servicemen with chronic periapical infection in comparison with servicemen with inflammatory processes in other parts of the face was observed. In the control group, the average value of CD4/CD8 was within normal limits (1.3-2.5), amounting to 1.46 . The difference was insignificant $(\mathrm{p}=0.12)$.

The average value of CD3+HLA-DR+in the main group were 1.3 times higher than normal $(0.5-6.0 \%)$, amounting to $7.6 \pm 5.0 \%$. In the control group, the average value of this indicator was within the norm, amounting to $5.6 \pm 3.1 \%$.

In contrast to CD3+HLA-DR+, the average value of CD3-HLA-DR+in the group of servicemen with periapical infection was within normal limits, amounting to $13.7 \pm 6.6 \%$. On the contrary, in the control group the value of this indicator exceeded the upper limit of the norm (16\%) by 1.17 times, amounting to $18.8 \pm 2.2 \%(\mathrm{p}=0.09)$ (Table 2).

Table 2

Average figures CD3-HLA-DR+(activated lymphocytes) of blood

\begin{tabular}{cccc}
\hline & \multicolumn{2}{c}{ Research groups } & p \\
\cline { 2 - 3 } Indicators (norm 0.5-6.0 \% & Main group $(\mathbf{n = 3 1 )}$ & Control group (n=5) & \\
\hline $\mathrm{M} \pm \mathrm{m}$ & $13.7 \pm 6.6$ & $18.82 .2 \pm$ & 0.09 \\
$\mathrm{Me}\left[\mathrm{Q}_{1} ; \mathrm{Q}_{2}\right]$ & $15[8.0 ; 18.0]$ & $18.0 ; 19.0]$ & $18.8[16.1 ; .5]$
\end{tabular}

Note: the variety of values in the main and control groups are statistically significant when $p<0.05$

Only one patient in the main group was with low CD38+values (below $36 \%$ ). High (above $50 \%)$ values of $\mathrm{CD} 38+$ were found in $51.6 \pm 8.9 \%(\mathrm{n}=16)$ patients of the studied main group and in $60 \pm 21.9$ - in the control group; the difference between the mean high values was insignificant $(\mathrm{p}=0.77)$ : respectively $52.8 \pm 5.9 \%$ vs. $60 \pm 21.9 \%$. The value of relative risk $(R R)$ in the main group was insignificant, amounting to $0.86[0.39 ; 1.9], \mathrm{p}>0.05$.

The correlation of interdependent indicators of cellular immunity of blood of patients in the main group was carried out (Table 3).

Table 3

Correlation ratios of lymphocytes in the main group $(n=31)$

\begin{tabular}{ccccc}
\hline \multirow{2}{*}{ Lymphocytes } & \multicolumn{2}{c}{ Direct correlation } & \multicolumn{2}{c}{ Inverse correlation } \\
\cline { 2 - 5 } & Lymphocytes & $\begin{array}{c}\text { correlation coefficient } \\
(\mathbf{p}<\mathbf{0 . 0 5})\end{array}$ & $\begin{array}{c}\text { Lymphocytes } \\
\text { correlation coefficient } \\
(\mathbf{p}<\mathbf{0 . 0 5})\end{array}$ \\
\hline CD 3 & & CD3-CD 16+56+ & -0.37 \\
CD 19 & CD3-HLA+ & 0.81 & & -0.56 \\
CD 4 & CD4/CD8 & 0.46 & CD 8 & -0.85 \\
CD 8 & & 0.87 & CD4/CD8 & -0.46 \\
CD3-CD 16+56+ & & & CD3+CD 16+56+ & -0.48 \\
CD3+CD 16+56+ & CD3+HLA-DR+ & & CD3+HLA-DR+ \\
CD3+HLA-DR+ & CD38+ & 0.39 & & \\
CD3-HLA+ & CD38+ & 0.36 &
\end{tabular}


As shown in Table 3, among military personnel hospitalized because of chronic periapical dental infection there is a direct correlation between six pairs of indicators and an inverse correlation - between five. Including the dense correlation, the coefficient was determined between CD 19+(B-lymphocytes) and CD3-HLA+; CD 4 i CD4/CD8 ( $r>0.70)$. The high inverse correlation was defined between CD 8 and CD4/CD8 ( $r<-0.70)$. 19+cells are an important part of the humoral immune response HLA-DR+- marker of late and prolonged cell activation. HLA-DR-positive lymphocytes circulate in the blood for a long time, and the expression of this marker most fully reflects the activation state of cells. CD3+CD4+- T-helpers are involved in the induction of a specific immune response. One of the important functions is the production of cytokines, which affect all parts of the inflammatory process, and can direct the immune response to a predominantly humoral or cytotoxic pathway and regulate its intensity. The correlation of interdependent indicators of cellular immunity of patients' blood in the control group is given in the Table 4.

Table 4

Correlation ratios of lymphocytes in the control group $(n=5)$

\begin{tabular}{ccccc}
\hline \multirow{2}{*}{ Lymphocytes } & \multicolumn{2}{c}{ Direct correlation } & \multicolumn{2}{c}{ Inverse correlation } \\
\cline { 2 - 5 } & Lymphocytes & $\begin{array}{c}\text { correlation coefficient } \\
(\mathbf{p}<\mathbf{0 . 0 5})\end{array}$ & $\begin{array}{c}\text { Lymphocytes } \\
\text { correlation coefficient } \\
(\mathbf{p}<\mathbf{0 . 0 5})\end{array}$ \\
\hline CD 3 & & CD38+ & -0.91 \\
CD 4 & CD4/CD8 & 0.89 & CD 8+ & -0.99 \\
CD 8 & & & CD4/CD8 & -0.95 \\
CD4/CD8 & CD3+HLA-DR+ & 0.39 & &
\end{tabular}

As it can be seen from Table 4 among servicemen hospitalized with inflammatory diseases of the maxillofacial area (except for chronic periapical dental infection), a dense correlation coefficient was determined in all interdependent indicators, except CD4/CD8 i CD3+HLA-DR+, where the correlation was weak $(\mathrm{r}<0.40)$.

\section{Discussion}

Our study gives an idea of the changes in the cellular link of immunity in chronic periapical infections of the military. The data obtained help to understand some of the reasons for the absence of clinical manifestations in the main group patients. In case of severe course of the periodontal disease, the number of CD8+cells increases, and the activity of peripheral blood cell killers also increase. In case of remission of CD3+, CD4+, CD8+, CD4+/CD8+, CD16, CD20 cells, functional activity of T-, V-lymphocytes and natural killer cells both in peripheral and in local blood flow corresponds the control parameters [11]. In our study, the average value of CD3+HLA-DR+in exacerbation of periodontitis were above normal (0.5-6.0 \%) 1.3 times, amounting to $7.6 \pm 5.0 \%$. Suppressive activity was more pronounced in exacerbation of chronic periodontitis in the military $(\mathrm{p}<0.05)$. The increase in CD3+above normal $(82 \%)$ in both the main and control groups could be taken as the lack of pronounced activation in the cellular immune system. The value of the relative sharpness (RR) of the risk of exacerbation of chronic periapical infection among servicemen with the same CD3+59.4\% was significant $1[0.2 ; 6.6], \mathrm{p}<0.05$.

According to the literary data, adaptive immunity was characterized by the presence of T-lymphopenia, mainly due to CD4+cells, with a slight increase in the number of CD8+cells, which was accompanied by the formation of a suppressor variant of immunodeficiency (the coefficient of immunoregulation $\mathrm{CD} 4 / \mathrm{CD} 8$ decreased against the same indicator of the reference rate by 1.66 times). In addition, the patients with chronic periodontitis had a significant increase in the number of $65 \mathrm{CD} 22+$ cells, with a decrease in the number of CD16+cells and their cytotoxic activity (the cytotoxicity index of the IC decreased relative to the reference rate by 1.22 times). In the presence of purulent-inflammatory complications in the observed patients, their indicators of cellular immunity had the greatest negative changes [12]. 
The regulatory functions of highly differentiated CD8+T cells may also include tissue repair [13], although this is mostly described in CD4+Treg for the lungs [14]. Indeed, experimental evidence for the existence of $\mathrm{CD} 8+\mathrm{T}$ cells endowed with tissue repair and/or bone regeneration in gum tissue is very scarce, as described below, requiring further research [15].

Of course, a comparative analysis of the results with clinical studies, as well as the study of the humoral part of the immune system would create a more complete picture of the peculiarities of the course of this pathology in the military. Despite this, the significance of this stage of our work is determined primarily by the novelty, namely, for the first time the task of studying the pathogenesis of chronic periapical infection without clinically pronounced signs of inflammation among ordinary servicemen. Difficulties in the research were associated with the peculiarities of the stay of patients in the service, which did not allow appointing them for repeated examinations and studying the features of the course of the disease in dynamics after discharge from the hospital. It should be noted that it is necessary to continue the study of immunity among military patients with no clinically expressed symptoms. The prediction and prevention of complications of periapical infection are especially important for people who are at military bases far from medical centers.

The theme provides a wide scope for further in-depth study of immune conditions in periodontitis, depending on individual indicators, such as age, location of the military unit and the general condition of the oral cavity before conscription among conscripts.

Study limitations. The measurements of the humoral immunity have not been studied which, in our opinion, is an important component for the pathology pathogenesis overview.

The perspectives of further research. It is planned to develop the algorithm for the correction of the cellular immunity state in order to determine the effect of apical parodontitis on their pathogenesis.

\section{Conclusions}

1. In this research we aimed at studying the indicators of the peripheral blood among the military patients suffering from periapical infections to confirm the relation between the infection in the periapical tissues and the consequent immune response to it.

2. As a result of the conducted research we found that the military patients who have chronic periapical infection, indeed suffer from immune suppression.

3. The properly conducted lab research confirmed that certain lymphocytes (T-helpers and B-lymphocytes) less actively participate in response to the chronic periapical infection than those of the control group.

4. According to the indicator of CD4+and CD 19+of patients' total blood with chronic periapical infection, it can be stated that there is a direct risk of exacerbation of chronic periapical infection among servicemen.

5. Response factors are as follows: CD8 and CD4 / CD8; CD19+(B-lymphocytes) and CD3HLA+; CD4 and CD4 / CD8 that again is a proof of the presence of the inflammatory process in the patients' organisms.

\section{Conflicts of interest}

The authors declare that they have no conflicts of interest.

\section{References}

[1] Nikolay, A. S., Sarbayeva, N. N., Dukal'skaya, O. Yu. (2010). Features of the response of the military personnel's immune system with low neuropsychic stability under the influence of military professional factors. Uchenyye zapiski, 2 (60), $116-118$.

[2] Vesna, Ye. A., Gulyuk, A. G. (2018). Biochemical analysis of oral salivary liquid, blood serum and pathologically changed periapical tissue according to inflammation activity parameters in patients with chronic apical periodontitis. Vestnik stomatologii, $3,15-20$.

[3] Kernits'kiy, R. V., Gulyuk, A. G., Goryanova, N. O., Ul'yanov, V. O. (2012). Features of morphofunctional changes in periodontal tissues in chronic periodontitis and periodontitis. Morfologiya, 4, 58-63.

[4] Sevast'yanov, A. V. (2016). Periodontitis. Etiology, pathogenesis, clinical picture, treatment. Saint Petersburg, 40. 
[5] Mikhalchenko, V. F., Manuylova, E. V., Yakovlev, A. T. (2012). Dynamics of indices of local immunity in the treatment of chronic periodontitis with the use of calcium-containing drugs. Biology and biotechnology, 1 (3), 37-40.

[6] Kanyura, O. A. (2014). The state of dental health and the need for orthopedic care for patients of a private dental institution. Bulletin of problems of biology and medicine, 4 (2), 279-282.

[7] Yugov, V. K. (2014). X-ray features of changes in periodontitis depending on the form and phase of the process. Radiology of the head and neck. Radiation diagnostics, radiation therapy, 4, 10-19.

[8] Könönen, E., Gursoy, M., Gursoy, U. (2019). Periodontitis: A Multifaceted Disease of Tooth-Supporting Tissues. Journal of Clinical Medicine, 8 (8), 1135. doi: http://doi.org/10.3390/jcm8081135

[9] Pashchenkov, M. V., Pinegin, B. V. (2002). The role of dendritic cells in the regulation of the immune response. Immunologiya, 5, 313-321.

[10] Pinegin, B. V., Yarilin, A. A. (2001). Application of flow cytometry to assess the functional activity of the human immune system. Moscow, 53.

[11] Nikolishin, A. (2012). Therapeutic dentistry. Vinnytsia: Nova Kniga, 680.

[12] Jin, L., Söder, B., Corbet, E. F. (2000). Interleukin-8 and Granulocyte Elastase in Gingival Crevicular Fluid in Relation to Periodontopathogens in Untreated Adult Periodontitis. Journal of Periodontology, 71 (6), 929-939. doi: http://doi.org/10.1902/ jop.2000.71.6.929

[13] Vasanthakumar, A., Kallies, A. (2015). The Regulatory T Cell: Jack-Of-All-Trades. Trends in Immunology, 36 (12), $756-758$. doi: http://doi.org/10.1016/j.it.2015.10.002

[14] Arpaia, N., Green, J. A., Moltedo, B., Arvey, A., Hemmers, S., Yuan, S. et. al. (2015). A Distinct Function of Regulatory T Cells in Tissue Protection. Cell, 162 (5), 1078-1089. doi: http://doi.org/10.1016/j.cell.2015.08.021

[15] Cardoso, E. M., Arosa, F. A. (2017). CD8+T Cells in Chronic Periodontitis: Roles and Rules. Frontiers in Immunology, 8. doi: http://doi.org/10.3389/fimmu.2017.00145 